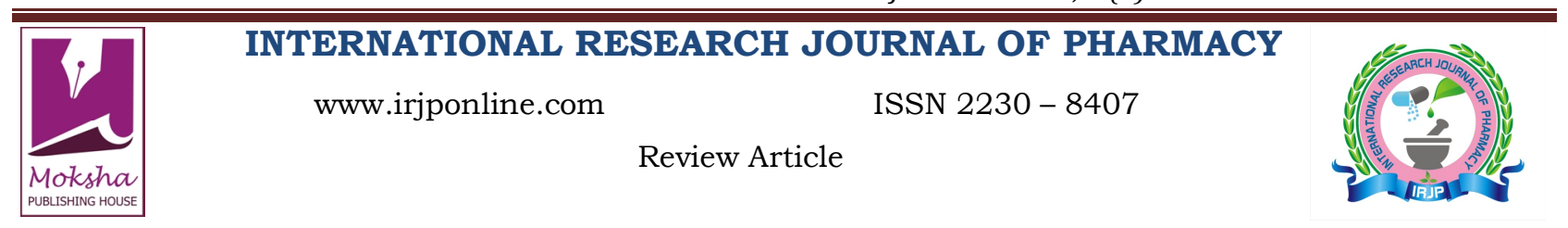

\title{
UPDATED INSIGHT ON FOAM BINDER GRANULATION
}

Saikh Mahammed Athar Alli*

Assistant Professor, Department of Pharmaceutical Sciences, Faculty of Health Sciences, Shiats, Allahabad, Uttar Pradesh, India

*Corresponding Author Email: atharodi@gmail.com

Article Received on: 20/08/13 Revised on: 01/09/13 Approved for publication: 10/09/13

DOI: $10.7897 / 2230-8407.04908$

IRJP is an official publication of Moksha Publishing House. Website: www.mokshaph.com

(C) All rights reserved.

\section{ABSTRACT}

To have updated handy reference as source of outstanding knowledge on foam binder granulation process in granulating the material(s). Granulation considered being important unit operation for producing pharmaceutical oral dosage forms. Revolutionising binder application methodology as advancement of wet granulation process best owed said process. It is a modified version of atomised spraying method and gaining wide acceptance. Product and process development calls for method of optimisation involving multidisciplinary activity and creates difficulties as several rudiments needed to be achievable, associated with umpteen features. Depth knowledge on process and formulation properties, monitoring granulation behaviour and its performance, is prerequisite. In this regard, information collected and presented as a handy note. Presented note will give insight on associated technicality and will assist researchers in getting optimised granule, having applicability in product evolution. This considered being offering outstanding knowledge and helping hand for getting granule with excellent feature.

Keywords: Binder, evolution, foam, granulation, process, technology.

\section{INTRODUCTION}

Granulation is a vital unit operation of pharmaceutical technology where small powdery particles agglomerated into larger entities called granule ${ }^{1}$. This executed for improving flow ability, appearance and mixing; reducing segregation; eliminating dustiness and detrimental properties; and improving physical or chemical properties of fine powders ${ }^{2-4}$. Granulation achieved through incorporation of binder (as solution or melt) onto powder or compressing them, to ensure their coalescence and growth ${ }^{1}$. This traditionally executed with wet-/dry-granulation process. Novel and modified processes includes fluidized-bed granulation, foam binder granulation (FBG), freeze granulation, melt/thermoplastic granulation, moisture activated dry granulation, pneumatic dry granulation, steam granulation, thermal adhesion granulation, and so on. All of these have limitations and superiority over each other ${ }^{4}$. Section of wet-granulation process calls for application of aqueous/non-aqueous solution of binder (SoB) and or polymer onto powder mass including instant release and controlled release products. Controlled release product uses release controlling polymers and in elevated quantity ${ }^{6}$. In production-scale, wet-granulation process uses spray technology for incorporating binders into powders, moving within granulator bowel. Type, geometry and location of nozzle, sprays geometry, spray rate, and droplet size influences performance of sprayer and hence efficiency of granulation process and granules ${ }^{7}$. The process of FBG developed abolishing many process-problems of sprayer in conventional spray method, considered substitute, and was acquiring wider acceptance ${ }^{8}$. FBG is advanced and refined method for incorporating binders in high-/low-shear granulator (HLSMG), extruder granulator, or fluid-bed processor (FBP), invented by The Dow Chemical Company (Midland, MI, USA). Said process designed to reduce complexity of spraying step and forefend plugging problems of spray nozzles ${ }^{9}$. Here aqueous SoB is added continuously or in batches to powdery particle bed in the form of foam, generated with foam generator ${ }^{9-12}$. Foam penetration involved in FBG method is less dependent on powder hydrophobicity comparing drop penetration, involved in spraying method ${ }^{13}$. It recognised as faster, simpler, and safer process. Being consider replacement for conventional atomised liquid spray technology gaining wider recognition ${ }^{14}$. Said process employs preferable and well recognised polymer (as binder) like hydroxypropylcellulose (KLUCEL ${ }^{\mathrm{TM}}$ ), ethyl cellulose (ETHOCEL ${ }^{\mathrm{TM}}$ ), poly(ethylene) oxide (POLYOX ${ }^{\mathrm{TM}}$ ), hydroxypropyl methylcellulose (hypromellose or METHOCEL ${ }^{\mathrm{TM}}$ ), hydroxypropylcellulose, methylcellulose, modified hydroxyethyl methyl cellulose (WALOCEL ${ }^{\mathrm{TM}}$ ), sodium carboxymethyl cellulose, and so on $^{11,14}$. Matrix system based controlled release tablets, instant release tablets, and disintegrating tablets formulated with said process. Several formulations successfully scaled up from laboratoryscale to pilot-scale with good process control ${ }^{9,10,15}$. In setting of above, strong need felt to focus updated information on FBG. Present works project information on background, issue, technicality, and current practice. Presented information has applicability in product evolution and predicting technical issues.

\section{Advantages}

- eliminates use of spray nozzle and eradicates plugging effects $^{10}$;

- accomplishes consistent binder distribution";

- demands low amount of binder for granulation;

- renders a low soak-to-spread ratio and achieves efficient binder delivery and particle coverage ${ }^{16}$;

- eradicates over wetting problem with diminished inprocess water requirement ${ }^{10}$;

- cost effective, fast, robust and reproducible processing ${ }^{10}$;

- lowers processing cost through reducing drying, processing, and equipment clean-up time. Besides eliminates need of attendant for attending spray nozzles, and all other variables like nozzle configuration, distance from moving powder bed, spray patterns, clogging, droplet size, and droplet distribution ${ }^{10}$;

- need not require new equipment or drastic changes in processing steps. Requires general equipments even in 
small-scale laboratory setting or full-scale production settings ${ }^{10}$;

- processes flexibility for adopting continuous or batch process $^{10}$

- yields lower proportion of coarse saturated lumps $(>2$ $\mathrm{mm}$ ) comparing spraying method, even during vigorous agitation of powder ${ }^{11}$;

- utilises proven and familiar water soluble binders like hydroxypropylcellulose, hypromellose, and so on ${ }^{10}$;

- immediate- and matrix-system based controlled-release, and orodispersible products can be easily scaled-up ${ }^{11,15}$; and

- Possess approval from several regulatory authorities ${ }^{3}$.

\section{Applications}

- $\quad$ suited for products with very low drug concentration ${ }^{10}$ or drug level (in $\mathrm{mg}$ or $\mu \mathrm{g}$ per tablet) resulting better drug distribution $^{13}$,

- able to carry active ingredients at a very low concentration or level with generated foam ${ }^{10,17}$,

- suited for very water-soluble or very poorly water soluble drugs and water sensitive formulations ${ }^{2}$, and

- Able in handling historically proven difficult materials including natural ingredients, the components of nutritional supplements ${ }^{3}$.

\section{Process and Technology}

FBG process enables granulation employing HLSMG, or twin-screw extruder (TSE) granulator, or FBP in both laboratory-scale and production-scale settings. This process involves step-batch, or batch, or continuous mode of adding SoB (as aqueous foam) onto previously blended powder bed contained in HLSMG ${ }^{9,10}$ or fluidized powder bed contained in FBP, kept in operation ${ }^{10,18,19}$. In TSE granulator, it applied through foamed binder application port, while in operation ${ }^{12}$. After attaining granulation endpoint wet mass is subsequently wet-milled. Resulting wet granules subsequently dried in FBP until attaining desired moisture content level ${ }^{9-12}$. Dried granules milled, sized, and characterised. Flow chart of FBG process is provided with Figure 1.

\section{Principle}

Conventional method of applying SoB involves encounter of spray droplet with a porous solid surface (powdery particle). This prevents its further travel from initial point of contact unless collision with another powder particle causes total/partial transfer of droplet onto the surface of colliding particle $^{3}$. In FBG SoB applied as foams. Applied foams are capable in flowing through porous media by the "breaking and remaking process". In the process of breaking and remaking bursting of foam bubbles may lead to bridging of lamellae and powder thus can regenerate foam as lamellae gets contacted by free gas or foam. This mechanism improves ability of foam to spread rather than to get soaked on encountering particles. Which in turn coats surface of powder, before absorption of liquid from the foam will be effective. As foams replace water with air, process possesses "low wet pickup property"13,16,20,21. Here, distribution of SoB throughout powder bed gets improve associated with tremendous increase in surface area of liquid and foam volume of polymeric binder. Generated liquid droplets (foam) from foamed SoB had a high spread-to-soak ratio thus got spread on the surface of particles to coat it and place binder where actually needed. This makes binder distribution more consistent ${ }^{5,9,10,22}$. In case of extruder mechanical dispersion dominated nucleation mechanism is involved ${ }^{12,13}$. The FBG process involves mechanical dispersion type of nucleation mechanisms that differs from spray which tends to incur early liquid penetration type of nucleation mechanisms $^{5,13}$.

\section{Foam Generation and Application}

Foam generator used, is a modified version of early design. Modification is incorporation of an air intensifier that intensifies and generates air pressure $\leq 150$ psi from in-house air supplies of 40-80 psi. Intensified air pressure enabling foaming of viscous fluids ${ }^{23}$. Laboratory-scale foam generator used for laboratory-scale and pilot-scale production, but heavy duty one used for manufacturing-scale ${ }^{9,10}$. Foamed binder pumped via a plastic pipe into granulator bowl at suitable location where the blades will not be foul by pipe. Said pipe simply inserted through any convenient port accessing in to granulator bowl. Pumping carried out following any one of three distinct modes (i.e., batch, stepbatch, and continuous) at a rate of 75 to $300 \mathrm{~g} / \mathrm{min}$, depending on product ${ }^{9,10}$.

\section{Quality of Foam}

Concentration, molecular weight, and substitution-type of the polymer impart greatest flexibility to nature of aqueous phase and foam quality (FQ), and monitor stability of foam. Requisite stability of foam is highly process dependent. Viscous solutions with viscosity 150-300 cP generally produce highly elastic foams having high yield stress. These foams tediously produced, often have lowest FQ, having very low tendency to spread. Low viscosity having viscosity of 3$100 \mathrm{cP}$ very conveniently foamed and can result very high quality foams with low density?

Foams quantified in several ways while FQ calculated from penetration time and nucleation ratio. Said values used to determine range of acceptable FQ needed to get granules with wished quality ${ }^{9,11,24-26}$. FQ is determined from volume of liquid (VLiq) and volume of gas (VGas) following the relation":

$$
\mathrm{FQ}=\mathrm{VGas} /(\mathrm{VGas}-\mathrm{VLiq}) \times 100 \text { at NTP }
$$

Higher FQ value indicates higher surface area. Improvement in surface area of foams improves binder efficiency and its potentiality to carry various components (like highly potent and low volume drugs) into powder mass. FQ presented from wet foam (FQ $<70)$ to dry foam (FQ $>70)$. Wet foam has physical characteristics and flow similar to a liquid and exhibit a low rheological yield stress. Dry foam has a high air-to-liquid ratio and moves like solid with comparatively high rheological yield stress. They exhibit solid-like flow properties below their yield stress, tend to flow in pipes by wall slippage, and recommended for granulation ${ }^{9,10}$. Increasing FQ through decreasing $\mathrm{SoB}$ concentration or liquid-to-solid ratio decreases average granule $\operatorname{size}^{27}$. Improvement in surface area of foam improves binding efficiency of binder with same liquid and liquid-to-air ratio. 


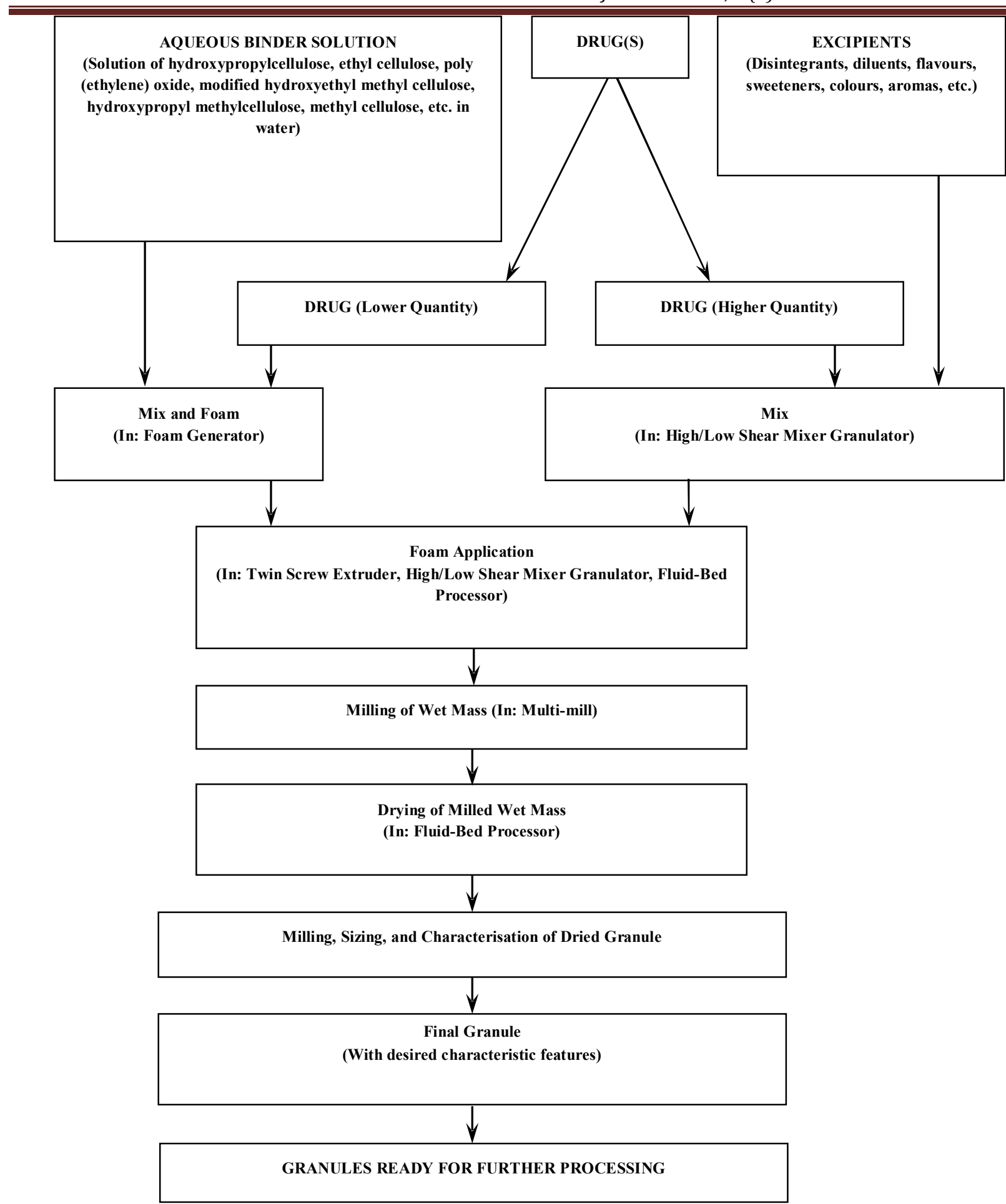

Figure 1: Flow Chart of Foam Binder Granulation Process 
Said parameter of foam calculated from thickness of foam lamellae $(\mathrm{H})$, surface area of foam $(\mathrm{A})$, and volume of liquid $\left(\mathrm{V}_{\text {Liq }}\right)$ using given relation?

$$
\mathrm{H}=2 \mathrm{~V}_{\mathrm{Liq}} / \mathrm{A}
$$

Foam density $\left(\rho_{\mathrm{F}}\right)$ readily calculated from density of liquid phase $\left(\rho_{\mathrm{Liq}}\right)$, volume of liquid $\left(\mathrm{V}_{\mathrm{Liq}}\right)$ and volume of foam $\left(\mathrm{V}_{\mathrm{F}}\right)$ employing given equation ${ }^{9}$.

$$
\rho_{\mathrm{F}}=\rho_{\mathrm{Liq}}\left(\mathrm{V}_{\mathrm{Liq}} / \mathrm{V}_{\mathrm{F}}\right)
$$

Value of FQ and foam density, and thickness of foamlamellae indicate foam stability. Stabilisation of foam is done with incorporation of non-aqueous liquids ${ }^{28}$ and solids ${ }^{29}$ in formula. Cellulose ethers were effective foam stabilisers enabling in preparing foams of various stabilities ${ }^{9}$.

\section{Penetration Kinetics of Foam}

Nucleation and binder distribution plays critical role in determining size distribution of granule in FBG processes ${ }^{21}$. Penetration and nucleation kinetics data of foam indicates its penetrability through loosely packed powdery particle bed. It is determined form specific penetration, penetration time and nucleation ratio. Value of nucleation ratio and penetration time is useful in quantifying liquid distribution efficiency and nuclei formation kinetics ${ }^{20,24-26,30}$. Specific penetration $\left(t_{\mathrm{ps}}\right)$ is penetration time $\left(t_{p}\right)$ per unit mass of binder $(m)$ while penetration time is time required for a drop or foam to penetrate completely into powder bed ${ }^{30}$. Value of $t_{p s}$ is invulnerable to variations in dispersed foam mass, a limitation of $\mathrm{t}_{\mathrm{p}}{ }^{30}$.

$$
\mathrm{t}_{\mathrm{ps}}=\mathrm{t}_{\mathrm{p}} / \mathrm{m}
$$

Nucleation ratio $\left(\mathrm{K}_{\mathrm{m}}\right)$ reflects distribution efficiency of binder through powder mass to create granule. It is amount of powder nucleated per unit mass of $\operatorname{SoB}^{16,20,24,30} . \mathrm{K}_{\mathrm{m}}$ calculated from ratio of nucleus mass $\left(\mathrm{M}_{\mathrm{n}}\right)$ to SoB mass $\left(\mathrm{M}_{\mathrm{b}}\right)$.

$$
\mathrm{K}_{\mathrm{m}}=\mathrm{M}_{\mathrm{n}} / \mathrm{M}_{\mathrm{b}}
$$

Foam's $t_{p}$ depends on formulation while roping powder flow and agitation is crucial in rapidly dispersing foam ${ }^{16} . \mathrm{K}_{\mathrm{m}}$ depends on foam addition method ${ }^{20}$. Drop penetration time decreases with decrease in proportion of hydrophobic component whereas hydrophobicity strongly influences same $^{30}$. Wettability of powder mixture had a pronounced affect on granule properties. Whilst hydrophobicity of it proportionately decreases granule strength and granule size ${ }^{30}$.

\section{Effect of Processing Parameters on Granule Size}

Flow rate and screw speed are most significant factors affecting particle size and granule fracture strength. Binder molecular weight had a minor influence on granule properties and FQ have insignificant influence when extruder is used ${ }^{12}$. Increasing $\mathrm{FQ}$, and decreasing concentration of bindersolution and liquid-to-solid ratio decreases average granule size, but effect is interrelated with impeller speed ${ }^{27}$. Foam delivery tends to create a narrower nuclei size distribution during early stage of wet granulation. Nuclei formation influenced by foam properties and powder flow conditions within granulator. Narrowest size distribution of nuclei obtained with high-quality foam and intensive powder mixing conditions. Coarser nuclei formed with low-quality foam and sketchy powder mixing condition ${ }^{27}$.

\section{FBG with the HLSMG}

\section{Small-scale laboratory setting}

In small-scale laboratory setting, granulation carried with a 10-L to 20-L HLSMG. Foamed binder delivered in to powder bed contained within HLSMG under running condition through port located on granulator lid. It dispersed in powder by operating side chopper blades between 300-1,800 revolutions per minute (RPM) and main blade speed between 30-300 RPM ${ }^{3}$. Step-batch mode of foamed binder addition involves addition of aliquot amount of foam to powder bed for specified time at preset liquid flow rate while granulator is in operation. Granulator run until foam got disperse homogeneously into powder bed. Then degree of wetness and granularity of granules examined visually and manually. Process of foamed binder addition and subsequent evaluation of granules for degree of wetness and granularity repeated until an acceptable wetness and granularity attained. Granules deemed to acceptable at a point when a compressed handful of granules holds loosely together and easily breaks over again into agglomerated particles ${ }^{3}$. Batch mode involves placing entire amount of foam on top of powder at any suitable flow rate before HLSMG set into operation. Continuous mode involves continuous addition of foam into powder bed while granulator is in operation. This is resulting homogeneous dispersion of foam in powder as added ${ }^{3}$. Damp mass had to be wet-milled employing multi-mill equipped with suitable screen, holes, and an impeller, operated at suitable speed. Granules air dried or dried with FBP at suitable temperature. Dried granules is sized using multi-mill with higher size screens while size of opening and speed of impeller tip to be kept same ${ }^{10,31,27}$. During initial stage of development step-batch mode followed and also for granulating unfamiliar powder system. It is also follow for determining relative proportions of foam and powder to be employing in successive batch or continuous granulation processes for processing of equivalent formulation.

\section{Pilot-scale setting}

Granulation in pilot-scale setting done with a $50-\mathrm{L}$ to $150-\mathrm{L}$ HLSMG operated with main blade speed of lower RPM and a chopper speed of same RPM, comparing small-scale settings. Foam added via a 0.5-1.0 inch tube through a porthole on lid of bowl, in continuous mode. The damp mass wet-milled employing multi-mill operated at similar operational conditions, comparing small-scale settings. Granules air dried or dried with FBP at similar operational conditions, comparing small-scale settings. Dried granules sized using multi-mill with higher size screens while size of opening and speed of impeller tip to be kept same ${ }^{10,11}$.

\section{Manufacturing-scale setting}

Granulation in manufacturing-scale setting can be done with a 500-L to 600-L HLSMG operated with main blade speed of lower RPM and a chopper speed of same RPM comparing pilot-scale settings. Foam added via a 1.5-2.0 inch tube through a porthole on lid of bowl, in continuous mode. The damp mass wet-milled employing multi-mill operated at similar operational conditions comparing pilot-scale settings. Granules air dried or dried with FBP at similar operational conditions comparing pilot-scale settings. Dried granules sized using multi-mill with higher size screens while size of opening and speed of impeller tip is kept same ${ }^{10}$. 


\section{FBG with TSE}

Here granulation of material done continuously between 20$40 \mathrm{~kg} / \mathrm{h}$ and 220-320 RPM using co-rotating TSE while controlling extruder barrel temperature to $25-40^{\circ} \mathrm{C}$ using a cooling system. Addition of binder fluid is done by pumping it, after foaming to $75-95 \%$ FQ using a mechanical foam generator, through injection port of extruder. Foamed binder metered accurately into extruder using a side stuffer (auxiliary feeder affixed to the extruder), at all operating conditions $^{12}$. Thermo Fisher Scientific Inc. (Waltham, USA) has several ranges of TSE granulators ranging from lab-scale to plant-scale (Pharma 11, Pharma 16 TSG and Pharma 24 TSG) ${ }^{4}$.

\section{FBG with FBP}

FBP used for granulating material using foamed binders ${ }^{10,18,19}$. Addition of binder fluid is done by pumping it (after foaming it to $75-95 \%$ FQ using a mechanical foam generator) through pipe that is used for spraying purpose after removing atomising nozzle assembly ${ }^{10}$. FBP based granulators ranging from lab-scale to plant-scale are available from several manufacturers ${ }^{4}$.

\section{Evaluation of Granules}

Granulations evaluated for loss on drying, flow property, density, and particle size and size distribution ${ }^{33}$; morphology by Scanning Electron Microscopy ${ }^{32}$; and surface area, median pore diameter (volume), total intrusion volume, and bulk density following mercury porosimetry method ${ }^{8}$.

\section{In vitro dissolution study}

In vitro drug release profile of granules studied with IP/BP/USP dissolution (basket type) apparatus operated at $37^{\circ} \mathrm{C} \pm 0.5^{\circ} \mathrm{C}$ and suitable RPM containing discriminatory dissolution medium. Granules kept in basket (wrapped with suitable cloth) of dissolution apparatus. Aliquot quantity of dissolution medium withdrawn at preset periods, and immediate replacement with equal volume of fresh one. Drug content in withdrawn sample analysed suitably and result presented in percent referring actual drug content value ${ }^{33}$.

\section{In vitro release kinetic study, statistical evaluation, and data fitting}

A mean value of three determinations at each time point used to fit in vitro drug release profile to different kinetic models for finding their release exponents, while the mean value of twelve determinations used for estimating difference factor, similarity factor, and two indices of Rescigno. Statistical analysis of percent released data and other data performed using one-way analysis of variance at a significance level of $5 \%$. In vitro release kinetic studies, statistical evaluation, data fitting, nonlinear least square curve fitting, simulation, and plotting done with suitable software for determining parameters of each equation $^{32,33}$.

\section{FBG Equipment}

\section{Laboratory-scale equipment}

Vertical high-shear granulator model FM-VG-10 (Glatt Air Techniques, Ramsey, NJ, USA), FBP based MP-MICRO ${ }^{\text {TM }}$ (GEA Process Engineering nv, Wommelgem, Belgium) and MIDI-LAB (DIOSNA Dierks and Söhne GmbH, Osnabrück, Germany); TSE based Pharma 11; and laboratory-scale foam generator (Corrosion Fluids Inc., Midland, MI, USA) ${ }^{4}$.

\section{Pilot-scale equipment}

Top-drive high-shear granulator model GMX-150 (Vector Corp., Marion, IA, USA); FBP based MULTIPROCESSOR $^{\text {TM }}$ (GEA Process Engineering nv), Pilot Fluid Bed Dryers/Granulators (Ohara Technologies Inc., Richmond Hill, Ontario, Canada) and Fluid Bed Processor-50 (Umang Pharmatech, Mumbai, India); TSE based Pharma 16 TSG; and a laboratory-scale foam generator (Corrosion Fluids Inc. $)^{4}$.

\section{Manufacturing-scale equipment}

Top-drive high-shear granulator (GEA Niro Pharma Systems, Columbia, MD, USA); FBP based Fluid Bed Processor 125/500/750/1000/1500 (Umang Pharmatech), Production Fluid Bed Dryers/Granulators or Multi Processor (Ohara Technologies Inc.); TSE based Pharma 24 TSG; and a production-scale foam generator model Top Mix type 100 (Hansa Industrie-Mixer GmbH and Co., Stuhr-Heiligenrode, Germany) $)^{4}$.

\section{CONCLUSION}

The FBG process is very controllable and results granules with acceptable properties. Better understanding of flow patterns, mixing behaviour and impact velocities in different types of granulation equipment also needed. FBG process can be successfully used to get diversified product including controlled-release and immediate-release granules of several drugs, including water sensitive formulations. Application of FBG technique by pharmaceutical and other industries will be a function of inherent conservatism and statutory limitations, a major challenge to overcome. Processing process, desired $\mathrm{FQ}$, processing equipment should be judiciously selected to get granules with desired characteristics and robustness.

\section{REFERENCES}

1. Gabriel IT. Preface. Powder Technol 2001; 117(1-2): 1-2.

2. Turton R, Bhatia A, Hakim H, Subramanian G, Norman L. Research in particle coating and agglomeration at West Virginia University. Powder Technol 2001; 117(1-2): 139-148. http://dx.doi.org/10.1016/S00325910(01)00325-4

3. Rios M. Foam granulation technology update and future applications. Pharm Technol. Available from: http://www.pharmtech.com/pharmtech/ Article/Foam-Granulation-Technology-Update-and-Future Appl/ArticleStandard/Article/detail/514845; 2008.

4. Saikh MAA. A technical note on granulation technology: a way to optimise granules. Int J Parm Sci Res 2013; 4(1): 55-67.

5. Iveson SM, Litster JD, Hapgood K, Ennis BJ. Nucleation, growth and breakage phenomena in agitated wet granulation processes: a review. Powder Technol 2001; 117(1-2): 3-39. http://dx.doi.org/10.1016/ S0032-5910(01)00313-8

6. Ritala M, Jungersen $\mathrm{O}$, Holm $\mathrm{P}$, Schæfer $\mathrm{T}$, Kristensen HG. A comparison between binders in the wet phase of granulation in a high shear mixer. Drug Dev Ind Pharm 1986; 12(11-13): 1685-1700. http:/ /dx.doi.org/10.3109/03639048609042603

7. Litster JD, Hapgood KP, Michaels JN, Sims A, Roberts M, Kameneni SK, et al. Liquid distribution in wet granulation: dimensionless spray flux. Powder Technol 2001; 114(1-3): 32-39. http://dx.doi.org/10.1016/ S0032-5910(00)00259-X

8. Sheskey P, Keary C, Balwinski K, Kenny R. Foam granulation technology: impact of manufacturing variables on final granule and tablet properties. Poster presented at the Annual Meeting and Exposition of the American Association of Pharmaceutical Scientists (AAPS). Atlanta, Georgia; 2008.

9. Keary CM, Sheskey PJ. Preliminary report of the discovery of a new pharmaceutical granulation process using foamed aqueous binders. Drug Dev Ind Pharm 2004; 30(8): 831-845. http://dx.doi.org/10.1081/DDC200030504 PMid:15521329

10. Sheskey P, Keary C, Inbasekaran P, Deyarmond V, Balwinski K. Foam technology: the development of a novel technique for the delivery of aqueous binder systems in high-shear and fluid-bed wet-granulation applications. Poster presented at the Annual Meeting and Exposition of 
the American Association of Pharmaceutical Scientists. Salt Lake City, Utah; 2003.

11. Sheskey P, Keary C, Clark D, Bahwinski K. Scale-up trials of foamgranulation technology -- high shear. Pharm Technol ( Europe) 2007; 19(9): 37.

12. Thompson MR, Weatherley S, Pukadyil RN, Sheskey PJ. Foam granulation: new developments in pharmaceutical solid oral dosage forms using twin screw extrusion machinery. Drug Dev Ind Pharm 2012; 38(7): 771-784. http://dx.doi.org/10.3109/03639045.2011.633265 PMid:22085462

13. Tan MX, Nguyen TH, Hapgood KP. Drug distribution in wet granulation: foam versus spray. Drug Dev Ind Pharm 2013; 39(9): 13891400. http://dx.doi.org/10.3109/03639045.2012.719233 PMid:23057532

14. Tan MXL, Hapgood KP. Foam granulation: binder dispersion and nucleation in mixer-granulators. Chem Eng Res Des 2011; 89: 526-536. http://dx.doi.org/10.1016/j.cherd.2010.07.001

15. Ahmed TA, El Say KM, Mahmoud MF, Samy AM, Badawi AA. Miconazole nitrate oral disintegrating tablets: in vivo performance and stability study. AAPS Pharm Sci Tech 2012; 13(3): 760-71. http://dx.doi.org/10.1208/s12249-012-9798-z PMid:22585373 PMCid:PMC3429679

16. Tan MXL, Wong LS, Lum KH, Hapgood KP. Foam and drop penetration kinetics into loosely packed powder beds. Chem Eng Sci 2009; 64(12): 2826-2836. http://dx.doi.org/10.1016/j.ces.2009.03.008

17. Flores R, Keary C, Sheskey P, Hitt J, Elder E, Rogers T. Advantages of foam granulation and process miniaturization for small quantities of micronized drugs. Poster presented at the Annual Meeting and Exposition of the American Association of Pharmaceutical Scientists. Nashville, Tennessee; 2005.

18. Maresca A, Ancilletta G. Process and apparatus for treating particalized substances in a fluidized bed. US Patent 3631608; 1972.

19. Victor JG. Fluidized bed apparatus and process. US Patent 4229289; 1980.

20. Tan MXL, Wong LS, Lum KH, Hapgood KP. Foam granulation: comparison of nucleation via drops and foams. Chemeca, Towards a Sustainable Australasia [Barton, ACT]; Engineers Australia; 2008. p. 372-381.

21. Tan MXL, Hapgood KP. Foam granulation: liquid penetration or mechanical dispersion? Chem Eng Sci 2011; 66(21): 5204-5211. http://dx.doi.org/10.1016/j.ces.2011.07.012

22. Koo OM, Ji J, Li J. Effect of powder substrate on foam drainage and collapse: implications to foam granulation. J Pharm Sci 2012; 101(4): 1385-1390. http://dx.doi.org/10.1002/jps.23053 PMid:22234920
23. Fry JF, French RJ. A mechanical foam-generator for use in laboratories. J Appl Chem 1951; 1(10): 425-429. http://dx.doi.org/10.1002/jctb. 5010011001

24. Schaafsma SH, Vonk P, Kossen NWF. Fluid bed agglomeration with a narrow droplet size distribution. Int J Pharm 2000; 193(2): 175-187. http://dx.doi.org/10.1016/S0378-5173(99)00329-4

25. Hapgood KP, Lister JD, Biggs SR, Howes T. Drop penetration into porous powder beds. J Colloid Interface Sci 2002; 253(2): 353-366. http://dx.doi.org/10.1006/jcis.2002.8527 PMid:16290866

26. Kariuki WIJ, Smith RM, Rhodes M, Hapgood KP. Prediction of liquid coverage in fluid bed granulation. Chemeca, Engineering at the Edge: 26-29; Engineers Australia; 2010. p. 1649-1660.

27. Tan MXL, Hapgood KP. Foam granulation: Effects of formulation and process conditions on granule size distributions. Powder Technol 2012; 218: 149-156. http://dx.doi.org/10.1016/j.powtec.2011.12.006

28. Arnaudov L, Denkov ND, Surcheva I, Durbut P, Broze G, Mehreteab A. Effect of oily additives on foam ability and foam stability. 1. Role of interfacial properties. Langmuir 2001; 17(22): 6999-7010. http://dx.doi.org/10.1021/la010600r

29. Bindal SK, Sethumadhavan G, Nikolov AD, Wasan DT. Foaming mechanisms in surfactant free particle suspensions. AIChE J 2002; 48(2): 2307-2314. http://dx.doi.org/10.1002/aic.690481020

30. Nguyen T, Shen W, Hapgood K. Drop penetration time in heterogeneous powder beds. Chem Eng Sci 2009; 64(24): 5210-5221. http://dx.doi.org/10.1016/j.ces.2009.08.038

31. Tan MXL, Hapgood KP. Foam granulation in a high shear mixergranulator. Chemeca, Engineering at the Edge, 26-29, Hilton Adelaide, South Australia [Barton, ACT]; Engineers Australia; 2010. p. 18261835 .

32. Alli SM. Preparation and characterization of a coacervate extendedrelease microparticulate delivery system for Lactobacillus rhamnosus. Int J Nanomedicine 2011; 6: 1699-707. PMid:21984867 PMCid: PMC3184930

33. Alli SMA, Samanta A, Mukherjee B, Ali SMA, Dehury G, Kanungo S. Hydrophilic polymeric matrix tablet for sustained delivery of levofloxacin. Int J Pharm Sci Tech 2010; 5(2): 40-55.

\section{Cite this article as:}

Saikh Mahammed Athar Alli. Updated insight on foam binder granulation. Int. Res. J. Pharm. 2013; 4(9):35-40 http://dx.doi.org/10.7897/2230$\underline{8407.04908}$ 